\title{
GLAD!
}

Revue sur le langage, le genre, les sexualités

$10 \mid 2021$

Varia

\section{Souhaits oubliés : documents de fin de vie des personnes trans vivant avec une démence aux marges des changements juridiques}

Forgotten Wishes: End-of-life Documents for Trans People with Dementia at the Margins of Legal Change

\section{Alexandre Baril, Marjorie Silverman, Marie-Claire Gauthier et Maude Lévesque}

Traducteur : Catriona LeBlanc

\section{(2) OpenEdition} Journals

Édition électronique

URL : https://journals.openedition.org/glad/2460

DOI : $10.4000 /$ glad.2460

ISSN : 2551-0819

Éditeur

Association GSL

Référence électronique

Alexandre Baril, Marjorie Silverman, Marie-Claire Gauthier et Maude Lévesque, « Souhaits oubliés : documents de fin de vie des personnes trans vivant avec une démence aux marges des changements juridiques », GLAD! [En ligne], 10 | 2021, mis en ligne le 01 juillet 2021, consulté le 26 juillet 2021. URL http://journals.openedition.org/glad/2460 ; DOI : https://doi.org/10.4000/glad.2460

Ce document a été généré automatiquement le 26 juillet 2021.

\section{(†)

La revue GLAD! est mise à disposition selon les termes de la Licence Creative Commons Attribution Pas d'Utilisation Commerciale - Pas de Modification 4.0 International. 


\section{Souhaits oubliés : documents de fin de vie des personnes trans vivant avec une démence aux marges des changements juridiques}

Forgotten Wishes: End-of-life Documents for Trans People with Dementia at the Margins of Legal Change

Alexandre Baril, Marjorie Silverman, Marie-Claire Gauthier et Maude Lévesque

Traduction : Catriona LeBlanc

\section{NOTE DE L'AUTEUR}

Les auteures et auteur tiennent à remercier la direction de ce numéro spécial, ainsi que la direction du Canadian Journal of Law \& Society/Revue Canadienne Droit et Société pour leur précieux soutien au cours du processus de publication. Cette recherche est financée par une subvention Savoir du Conseil de recherches en sciences humaines (CRSH), et nous remercions cet organisme pour son généreux soutien financier.

Source originale : Baril, A., M. Silverman, M.-C. Gauthier et M. Lévesque (2020). « Forgotten Wishes: End-of-life documents for trans people with dementia at the margins of legal change ", Canadian Journal of Law \& Society / Revue Canadienne Droit et Société, Volume 35, no. 2, p. 367-390. doi:10.1017/cls.2020.13 


\title{
La démence et la planification préalable des soins : se préparer à tout oublier... même son identité de genre ${ }^{1}$
}

\begin{abstract}
Des prestataires de service dans un établissement de soins de longue durée ont partagé en entretien l'histoire d'Edna [...] Edna avait fait sa transition d'homme à femme 40 ans plus tôt. Avant son admission, le fils d'Edna lui avait présenté un ultimatum : soit elle se présentait comme homme, soit elle ne verrait plus jamais ses petits-enfants. [...] Edna s'y est soumise et ce n'est qu'au moment où le personnel l'a aidée dans la douche que son identité de femme transgenre a été découverte. Les prestataires de services, sur qui Edna dépendait pour défendre ses intérêts, ne savaient pas comment se confronter à la famille d'Edna et s'inquiétaient qu'une telle confrontation puisse compromettre son accès à ses petits-enfants. La démence d'Edna avait comme conséquence la perte de sa capacité à renseigner le personnel sur ses besoins, ce qui la rendait plus vulnérable aux demandes transphobes de sa famille. Elle dépendait de personnes qui ne comprenaient pas suffisamment ses besoins transgenres et elle était incapable de se défendre ellemême. [...] La présentation d'Edna comme homme pourrait être mal interprétée comme indication que son statut de personne trans s'était perdu en raison de sa démence. Mais cela n'était pas le cas. Sa démence a plutôt permis à sa famille de réaffirmer ses croyances transphobes, avec des conséquences dévastatrices. (Barrett et al. 2016 : 103-104)
\end{abstract}

1 La période de fin de vie, qui s'étend normalement sur les quelques mois précédant la mort, mais que nous définissons plus largement comme une période prolongée pendant laquelle une personne se situe à la fin de son parcours de vie plutôt qu'au début, est vécue différemment par divers groupes sociaux, dont les personnes âgées trans ${ }^{2}$. Le récit d'Edna, tel que décrit par le personnel de santé à l'équipe de recherche de Barrett et al. dans leur étude sur le vieillissement trans (2016), traduit les réalités difficiles auxquelles font face les personnes âgées trans vivant avec une démence ${ }^{3}$. Cette histoire met aussi en relief certains des débats actuels les plus controversés au sein des communautés trans, notamment la question de la « détransition ", c'est-à-dire l'acte de ne pas poursuivre une transition de sexe/genre déjà initiée ou de revenir au sexe/genre assigné à la naissance (Baril et Silverman 2019). Bien que la majorité des travaux sur la " détransition » se concentre sur la jeunesse trans, ce qui reflète la présence d'un biais âgiste plus général dans les études trans (McGovern 2014 ; Siverskog 2015 ; Toze 2018), un nombre surprenant d'écrits dans le domaine limité mais croissant sur les personnes âgées trans aborde les cas de "détransition » en lien avec la démence (e.g. Withall 2014 ; Latham et Barrett 2015a; Marshall, Cooper et Rudnick 2015; Knauer 2016; Witten 2016). À la fois les jeunes et les personnes âgées vivant avec des handicaps cognitifs constituent des groupes chez qui la capacité juridique ${ }^{4}$ est soit absente soit remise en cause (de Boer et al. 2010 ; Flynn 2018). Alors que certains travaux, dont ceux de Barrett et al. (2016) cités plus haut, semblent sceptiques quant à la "confusion» relative à l'identité de genre ou au désir de "détransitionner » et proposent que ces phénomènes s'expliquent par des pressions externes, d'autres travaux, fondés sur quelques cas anecdotiques présentés dans la littérature, concluent que la démence et ses changements associés pourraient avoir une incidence sur l'identité et l'expression de genre. Que ces écrits soutiennent ou pas l'idée de la "confusion » potentielle à l'endroit de l'identité de genre, ils suggèrent diverses stratégies d'intervention auprès de personnes trans vivant avec une démence (ci-dessous PTVAD) (e.g. Marshall, Cooper et Rudnick 2015 ; Peel et McDaid 2015 ; Hunter, Bishop et Westwood 2016 ; Westwood 2016 ; McFadden, Frankowski et Witten 2017 ; Baril et Silverman 2019). 
2 Les débats entourant la "détransition » ont suscité des réactions à la fois dans la littérature grise et scientifique quant aux stratégies pour protéger les droits des personnes trans en fin de vie, particulièrement les droits des personnes vivant avec des maladies et des handicaps, dont la démence. Louis Bailey (2012), par exemple, suivant son enquête sur les peurs et les préoccupations liées au vieillissement chez les adultes trans, conclut en suggérant cinq principes de pratiques exemplaires, notamment l'importance de la planification préalable en cas d' incapacité » juridique :

Planification préalable: On devrait encourager les personnes trans à créer un testament de vie et à écrire des directives claires pour leurs familles et les prestataires de soins, au cas où elles deviendraient incapables de prendre soin d'elles-mêmes. On devrait aussi conseiller aux personnes trans de nommer un individu qui est sensible à leurs besoins et qui est bien placé pour défendre leurs intérêts, au cas où elles développeraient une démence ou un trouble similaire, ce rôle peut être formalisé à l'aide d'une procuration perpétuelle. (Bailey 2012 : 36)

3 Comme nous le démontrons dans le présent article, les institutions et les organisations communautaires encouragent aussi les personnes trans à assurer la planification (juridique) de fin de vie ${ }^{5}$, y compris la préparation de documents de fin de vie, par exemple les testaments, la procuration et les directives préalables de soins pour guider la personne nommée comme décisionnaire autorisée dans la prise de décisions en cas d'« incapacité » juridique. Cet intérêt pour les préparatifs de fin de vie se manifeste au même moment qu'une transformation juridique profonde des réalités trans au Canada où, après une négligence historique, de nombreux changements se réalisent, notamment la protection offerte par les lois sur les droits de la personne, l'ajout de la désignation $\mathrm{X}$ sur les documents gouvernementaux, l'abrogation de l'exigence de subir une chirurgie pour changer officiellement sa désignation de sexe et un meilleur soutien pour le changement légal de sa désignation de sexe et de nom (Singer 2019 ; 2020). Pourtant, malgré ces réformes juridiques, les obstacles juridiques entravent toujours diverses sphères du quotidien des personnes trans. Le rapport TRANSforming JUSTICE : Trans Legal Needs Assessment Ontario (TRANSformer la justice: évaluation des besoins juridiques des personnes trans en Ontario) conclut que « les personnes trans ayant répondu au sondage indiquent un taux nettement plus élevé de problèmes juridiques justiciables par rapport à la population canadienne adulte » (James et al. $2018: 2$ ).

4 Tout en reconnaissant la valeur d'une approche juridique aux défis auxquels sont confrontées les PTVAD, nous soutenons qu'encourager les personnes trans à effectuer une "planification préalable" sous forme de documents (juridiques) de fin de vie repose souvent sur deux postulats non problématisés. Premièrement, l'identité et l'expression de genre semblent être comprises comme étant statiques, ou ce que Baril et Silverman (2019) nomment une "approche trans-affirmative stable» («transaffirmative stable approach ») selon laquelle "l'identité de genre est comprise comme étant une composante identitaire stable défaite par la démence, surtout dans un contexte cisgenriste qui ne soutient pas l'identité choisie » (Baril et Silverman 2019 : 6) ${ }^{6}$. Allant dans le même sens que leur critique de l'«approche trans-affirmative stable ", nous soutenons que la tentative d'assurer les droits des personnes trans à l'aide de documents (juridiques) de fin de vie laisse peu de place à la fluidité, à la reconfiguration ou à l'évolution du genre. Deuxièmement, l'appel aux personnes trans à assurer leurs droits par le biais de documents (juridiques) de fin de vie est fondé sur un postulat capacitiste/saniste/cogniticiste ${ }^{7}$ implicite selon lequel le soi pré-démence est mieux placé pour prendre des décisions que le soi qui vit avec une démence. 
5 À titre d'universitaires en sciences sociales adoptant une perspective interdisciplinaire, notre objectif dans le présent article est de nourrir les réflexions des juristes en mobilisant nos expertises en études trans, en gérontologie sociale et en études critiques sur la démence. En problématisant les deux postulats susmentionnés, nous démontrons que la solution proposée par plusieurs universitaires, activistes et par le personnel de soins pour gérer les défis associés à la démence chez les personnes trans âgées, particulièrement la préparation minutieuse de documents (juridiques) précisant leurs désirs, est fondée sur une analyse non intersectionnelle ${ }^{8}$ où la continuité biographique (Bury 1982; Sabat et Harré 1992 ; Sandberg 2018) est privilégiée à la fois en termes d'identité/expression de genre et de capacité cognitive. Dans de tels documents (juridiques), la potentielle fluidité du genre est niée, car l'identité/expression de genre sont comprises comme étant fixes. Ceci se produit peu importe que l'identité/ expression de genre énoncée soit masculine, féminine, les deux, ni l'une ni l'autre ou encore fluide, étant donné que, paradoxalement, une personne non binaire aurait à fixer sa non-binarité. En ce qui concerne la capacité cognitive, la continuité biographique se manifeste dans une plus grande valorisation des décisions prises par une personne vue comme étant « capable » sur le plan cognitif que celles prises par une personne vivant avec une démence. À partir de notre perspective intersectionnelle, nous critiquons les dimensions cisnormatives ${ }^{9}$ et cogninormatives ${ }^{10}$ des documents (juridiques) de fin de vie qui privilégient la cohérence et la continuité biographique.

6 Afin de démontrer cette thèse, nous mobilisons plusieurs champs d'études et cadres théoriques allant des études trans et de genre jusqu'aux études sur le handicap, crip ${ }^{11}$, de la folie (mad studies) et sur la démence. Notre cadre théorique est composé de concepts philosophiques, à savoir l'injustice épistémique (dont les injustices testimoniales et herméneutiques, termes forgés par Fricker, [2007]), la violence épistémique et la mort épistémique, ainsi que la résistance épistémique (Medina 2012 ; 2017). Bien qu'il semblerait que les réalités difficiles des PTVAD abordées dans cet article ne concernent qu'un nombre limité d'individus, Witten (2016) estime qu'il y a entre 4 et 12,3 millions de personnes trans âgées de plus de 65 ans qui pourraient développer une démence. De plus, nous espérons que ces réflexions amorceront des discussions fructueuses entre les universitaires et les personnes œuvrant dans une multitude de domaines d'études et de pratiques et qu'elles bénéficieront à toute personne qui prépare sa fin de vie, qu'elle soit handicapée ou pas, cis ou trans, jeune ou âgée.

7 Dans la section qui suit, nous abordons la littérature sur le vieillissement trans, ainsi que celle sur le vieillissement trans et la démence, puis démontrons en quoi les personnes trans sont déjà désavantagées lorsqu'elles tentent d'exercer les droits juridiques dont elles sont censées jouir. Nous présentons ensuite un cas hypothétique d'une PTVAD pour souligner les réalités complexes et les diverses formes d'oppression, c'est-à-dire le cisgenrisme et le cogniticisme, ayant un impact sur cette population. Nous mobilisons le concept d'injustice épistémique pour analyser cette étude de cas en particulier et les expériences des PTVAD en général. Pour conclure, nous proposons une approche intersectionnelle critique de la continuité biographique sur laquelle les documents et la planification (juridiques) de fin de vie dépendent. Cette analyse intersectionnelle, fondée sur une perspective trans-affirmative et positive envers les réalités crip et les personnes âgées, vise à fournir des solutions conceptuelles et concrètes aux dilemmes abordés dans cet article. 


\section{Personnes trans âgées vivant avec une démence : une réalité oubliée?}

8 Alors que la population canadienne vieillit rapidement et que l'incidence de la démence augmente, un nombre croissant d'individus au pays pourraient être jugés " incompétents », ce qui les rend plus vulnérables à diverses formes d'exclusion, de discrimination, de réduction au silence et d'exploitation. Bien que les débats entourant les diverses conceptualisations de l'«incompétence» et de la capacité juridique dépassent la portée du présent article, il est à noter que, comme l'observent Bach et Kerzner (2010), la notion de capacité au Canada est souvent « définie en relation avec la capacité de comprendre les informations pertinentes à la prise de décision et la capacité de reconnaître les conséquences raisonnablement prévisibles de prendre ou de ne pas prendre une décision. Dans ce sens, la "capacité" fait référence aux conditions cognitives perçues comme étant nécessaires à l'exercice de son droit à la capacité juridique [...] » (Bach et Kerzner 2010 : 16-17). Autrement dit, la « capacité » est fondée sur l'aptitude d'une personne à comprendre des informations sur le plan cognitif et à prendre des décisions et non pas sur la définition plus large de ce terme utilisée dans le domaine international des droits de la personne, qui «décrit la capacité juridique comme étant caractérisée par deux composantes : "la capacité de jouir d'un droit et la capacité d'agir et d'exercer ce droit [...]" » (Bach et Kerzner $2010: 16-17)$. Bach et Kerzner (2010) cherchent à interroger, comme nous le faisons dans cet article, les postulats souvent capacitistes derrière un emploi plus restrictif de la notion de capacité juridique, qui peut exclure certaines personnes vivant avec un handicap cognitif (Flynn 2018). Dans le cas des PTVAD, ce capacitisme constitue une des composantes d'un tableau de discriminations plus large, car les personnes trans font face à de nombreux mauvais traitements et de multiples obstacles lors de l'accès aux services sociaux et de santé (Ansara 2015 ; Hughes 2016 ; Kattari et Hasche 2016) et sont surreprésentées dans les statistiques sur la pauvreté, les enjeux de santé mentale et l'exclusion/l'isolement (Namaste 2000; Bauer et al. 2009; Bauer et Scheim 2015). Il convient de noter que toutes ces formes de discrimination et de violence sont étroitement liées aux déterminants sociaux de la santé pouvant mener les personnes trans à courir un plus grand risque de développer une démence (Bailey 2012; Hulko 2016; Westwood 2016; Witten 2016).

Ces déterminants sociaux de la santé, ainsi que plusieurs autres enjeux, sont explorés dans les écrits sur le vieillissement LGBTQ (Ward, Rivers et Sutherland 2012; Witten et Eyler 2012 ; Kia 2015 ; King et al. 2019). Toutefois, dans cette littérature de plus en plus abondante, les publications qui abordent la démence sont plutôt rares. Cette littérature, à la fois grise (Peel et McDaid 2015) et scientifique (Newman et Price 2012 ; McGovern 2014 ; Adelman 2016; Barrett et al. 2016; Hughes 2016; King 2016; Knauer 2016; Newman 2016; Ward et Price 2016; Westwood et Price 2016; Hogan 2017), se penche sur les réalités quotidiennes particulières des personnes LGBTQ vivant avec une démence, notamment dans les milieux institutionnels. Néanmoins, malgré l'intégration $\mathrm{du}$ « $\mathrm{T}$ » dans l'acronyme LGBTQ, la majorité de ces écrits ignorent les réalités distinctes des personnes trans pour se concentrer sur les communautés LGBQ (Namaste 2000; Ansara 2015; Baril 2019; de Vries et al. 2019). Par conséquent, les réalités trans sont mieux abordées dans la littérature limitée, mais grandissante, sur le vieillissement 
trans. Ces écrits scientifiques pionniers examinent principalement les formes de discrimination que vivent les personnes trans lors d'interactions avec le personnel de soins de santé et les pratiques exemplaires pour servir cette population (Cook-Daniels 2006 ; Persson 2009; Bailey 2012; Finkenauer et al., 2012; Hébert Chamberland et Chacha Enriquez 2012; Witten 2014; Fabbre 2015; Siverskog 2015; Ansara 2015; Latham et Barrett 2015a ; 2015b; Toze 2018; Pearce 2019; Pang Gutman et de Vries, 2019). Même si certains de ces travaux sont empiriques, d'autres publications demeurent uniquement théoriques, centrées sur une seule étude de cas ou fondées sur des données recueillies auprès d'adultes trans de tout âge sur leurs peurs associées au vieillissement, ce qui laisse peu de place aux voix des personnes trans âgées. Il en va de même pour le peu de textes abordant spécifiquement les personnes trans et la démence, et ce, à la fois dans la littérature grise (Alzheimer's Australia 2014; McFadden, Frankowski et Witten 2017) et la littérature scientifique (Marshall, Cooper et Rudnick 2015 ; Hunter, Bishop et Westwood 2016; Westwood 2016; Witten 2016 ; Baril et Silverman 2019). Il est surprenant de noter que, presque sans exception, la littérature sur les personnes LGBTQ et la démence discute de la « détransition » et de la « confusion » de genre.

10 À quelques exceptions près, dont Siverskog (2015), Hulko (2016), Wilson, Kortes-Miller et Stinchcombe (2018) et King et al. (2019), qui adoptent une perspective intersectionnelle, cette littérature n'aborde pas les liens précis entre les multiples identités des PTVAD et les conséquences des oppressions imbriquées. Cette lacune est surprenante, car, de notre point de vue, les expériences des PTVAD ne peuvent pas être comprises à partir d'une perspective ancrée uniquement dans les études trans, dans la gérontologie sociale ou dans les études critiques de la démence. Lorsque l'on demande en entrevue aux personnes trans de tous les âges de parler de leur vision, de leurs craintes et de leurs préoccupations relatives à l'avenir, elles se disent particulièrement concernées par les soucis de santé en général et les handicaps cognitifs en particulier. Elles craignent de devenir de plus en plus vulnérables et incapables de protester si elles sont incorrectement genrées ou subissent des microagressions (Stein et Almack 2012; Alzheimer's Australia 2014 ; Witten 2014 ; 2017 ; McFadden, Frankowski et Witten 2017 ; Almack 2019). D'ailleurs, dans le sondage MetLife sur la préparation et les perceptions face au vieillissement des personnes s'auto-identifiant comme transgenres (TMLS: Trans MetLife Survey on Later-Life Preparedness and Perceptions in Transgender-Identified Individuals) mené auprès de 1963 personnes, Witten (2016) rapporte que celles-ci craignaient spécifiquement le vieillissement, et plus particulièrement le handicap, la dépendance envers autrui et le développement d'une démence.

11 Pour les personnes trans, ces préoccupations et ces craintes, ancrées dans de nombreuses expériences de discrimination et de violence (Persson 2009; Siverskog 2015 ; Hughes et Cartwright 2015 ; Chamberland et al. 2016; Wilson, Kortes-Miller et Stinchcombe 2018; de Vries et al. 2019; Pang, Gutman et de Vries 2019) et dans quelques cas anecdotiques de "détransition", ont amené plusieurs auteur-es et organismes communautaires à souligner l'importance de bien se préparer à l'éventuelle possibilité de vivre un handicap cognitif, notamment en assurant la reconnaissance juridique de personnes soignantes désignées. De telles recommandations et initiatives pour les personnes LGBTQ se trouvent dans de nombreux guides pratiques comme ceux produits par Cartwright (2011), le National End of Life Care Programme (2012) et le National Resource Center on LGBT Aging (2014), ainsi que dans des articles et d'autres documents sur les questions de fin de vie pour les personnes LGBT, comme dans les 
travaux de Cook-Daniels (2006), Witten (2014), Hughes et Cartwright (2015), Chamberland et al. (2016), Knauer (2016), Cartwright et al. (2018) et de Vries et al. (2019).

12 Avant de passer dans la section qui suit à l'analyse des postulats cisnormatifs et cogninormatifs qui sous-tendent les documents et la planification (juridiques) de fin de vie, nous terminons la présente section en soulignant le fait que la préparation de documents juridiques de fin de vie demeure souvent inaccessible pour la plupart des personnes trans. Bien que certains documents juridiques, dont les directives préalables de soins, peuvent être préparés sans soutien juridique et même si le gouvernement canadien tente de renseigner la population sur le fait qu'il est possible de préparer ces documents sans aide juridique (Health Law Institute 2020), plusieurs personnes ont tout de même besoin de tels conseils. Sachant que les personnes trans vivent de multiples formes d'oppression, ainsi que des discriminations économiques comme le chômage et l'itinérance (Namaste 2000; Serano 2007; Bauer et Scheim 2015; Baril 2019), elles ne possèdent souvent pas les ressources financières nécessaires pour payer pour la préparation de ces documents (juridiques), ni un accès fiable aux expertises de notaires, de juristes ou d'autres personnes expertes qui connaissent les enjeux trans et font preuve de respect envers les personnes trans. Un tel accès aiderait à faire de la préparation de ces documents une expérience positive et sécuritaire (James et al., 2018). De plus, dans un contexte où plusieurs personnes trans luttent tout simplement pour survivre, la planification de fin de vie perd en importance (de Vries et al. 2019; Pang, Gutman et de Vries 2019). Wilson, Kortes-Miller et Stinchcombe (2018) nous rappellent aussi que les documents juridiques sont complexes et difficiles à comprendre et à utiliser. Pour terminer, malgré les recommandations et les incitatifs pour préparer les documents (juridiques) de fin de vie, un grand nombre de personnes trans demeurent peu préparées et sans accès au soutien et aux informations nécessaires pour faciliter leur processus de planification.

\section{À l'intersection de l'âgisme, du cisgenrisme et du cogniticisme : une étude de cas analysée sous l'angle des injustices épistémiques}

13 Comme cela a été mentionné plus tôt, plusieurs universitaires, institutions et organismes communautaires encouragent les personnes trans à préparer des documents (juridiques) précisant leurs souhaits quant à leur traitement en cas d'« incapacité ». Par exemple, le National Resource Center on LGBT Aging a publié un document intitulé Créer des documents de fin de vie pour les personnes trans: guide à l'intention des personnes qui défendent les droits trans (Creating End-of-Life Documents for Trans Individuals: An Advocate's Guide) (2014) qui invite les personnes trans à indiquer leurs préférences en matière de prénoms chosis, de pronoms et d'apparence physique. L'intention est que ces préférences guident les décisions prises en leur nom par leur décisionnaire. Cette organisation propose le formulaire suivant :

Pendant toute période de traitement, je demande à tout le personnel médical (médecins et autres) de m'appeler indépendamment de si j'ai

obtenu ou non un changement de nom au plan légal, de si j'ai changé ou non mon marqueur de genre sur quelque document ou de si j'ai reçu ou non quelconque traitement médical associé à ma transition de genre. Pendant toute période de 
traitement, je demande à tout le personnel médical (médecins et autres) d'utiliser les pronoms en parlant de moi, de mon dossier médical, de mon traitement, etc. [...] Pendant toute période de traitement, si je suis incapable de maintenir mon apparence _-_-__-__-__-_ par moi-même, je demande à tout le personnel médical (médecins et autres) de la maintenir dans une mesure raisonnablement possible [...] (National Resource Center on LGBT Aging 2014 : 2-3)

Certaines personnes pourraient soutenir que les dispositions et les documents juridiques ne sont pas pertinents pour la majorité des interactions routinières « mineures », étant donné que les directives préalables de soins couvrent normalement les décisions "majeures", comme le consentement à certains traitements médicaux. Parallèlement, bien que les directives puissent inclure une liste de valeurs et de principes pour orienter les décisionnaires, elles n'obligent pas le personnel de soins à effectuer certaines actions, par exemple, la provision de vêtements précis ou l'utilisation de pronoms particuliers. Certaines personnes pourraient donc douter de la pertinence des documents juridiques dans le contexte des formes d'interactions genrées examinées dans le présent article. Nous soutenons que, pour bon nombre de personnes trans, les interactions quotidiennes impliquant les pronoms, les vêtements, etc., constituent des éléments essentiels de la vie. Comme groupe, les PTVAD occupent une place particulièrement vulnérable où les soins personnels quotidiens peuvent devenir un lieu de plusieurs formes de discrimination. Nous reconnaissons que les documents juridiques disposent d'un pouvoir limité pour obliger le personnel soignant à interagir avec les PTVAD d'une certaine manière. Il est donc important pour les personnes trans de réfléchir à leur choix de décisionnaire afin de s'assurer que cette personne est trans-affirmative ou a été sensibilisée aux enjeux trans.

es individus tant trans que cis sont encouragés par une variété d'associations à mettre à jour les documents (juridiques) de fin de vie, au besoin, afin d'en assurer leur pertinence continue. L'Ontario Medical Association (OMA), par exemple, dans un document intitulé Planification préalable des soins: fiche d'information. Stratégie de soins de fin de vie de l'OMA (Advance Care Planning: Backgrounder. OMA's End-of-Life Care Strategy) (2014), affirme : «L'Ontario Medical Association (OMA) croit qu'il est important pour les individus de communiquer leurs volontés de fin de vie, quelles qu'elles soient. Les données indiquent que les préférences de soins d'un-e patient-e changent au cours du temps et en fonction des transformations de son statut de santé et de ses circonstances personnelles. Ceci souligne l'importance de concevoir la planification préalable des soins comme un processus continu et non pas comme un plan statique. »

16 Bien que nous soyons tout à fait d'accord avec l'OMA que la planification préalable des soins devrait être conçue comme un "processus continu ", en réalité, ces documents deviennent, pour reprendre les mots de l'OMA, un « plan statique » du moment qu'une personne est jugée juridiquement « incompétente ». Les documents juridiques peuvent changer, évoluer et être révisés jusqu'à ce qu'une personne perde sa capacité juridique, après quoi sa volonté exprimée dans ce plan devient immuable (Diller 2016). Alors que nous reconnaissons que la capacité juridique varie, théoriquement, selon les circonstances et les décisions ${ }^{12}$, en pratique, à partir du moment où une personne vivant avec une démence est jugée avoir perdu sa capacité décisionnelle dans un contexte quelconque, plusieurs membres du personnel soignant et de la famille interagissent avec celle-ci comme si elle avait perdu cette capacité dans tous les contextes. Pour le dire autrement, il semble y avoir un décalage entre la loi et l'application de cette loi. Selon des réflexions présentées en études critiques sur la 
démence (Bartlett et O'Connor 2010; Boyle 2014; Thomas et Milligan 2018), les représentations négatives des personnes vivant avec une démence tendent à jeter un discrédit supplémentaire sur leur capacité décisionnelle. Conséquemment, leur capacité juridique n'est pas régulièrement réévaluée, ce qui la rend «statique » au lieu de variable. L'établissement de la capacité juridique est étroitement lié aux débats philosophiques et éthiques sur le soi et l'identité individuelle (personhood) qui remontent aux années 1980. Même si l'espace nous manque dans cet article pour aborder les débats autour de l'identité individuelle et de la compétence juridique avant et après le développement de la démence ${ }^{13}$, il est important de noter que les positions dans ces débats sont extrêmement variées, allant de la conception d'une personne vivant avec une démence comme une personne entièrement "nouvelle " avec ses nouveaux besoins à respecter (e.g. Parfit 1984; Dresser 1995 ; Jaworska 1999) jusqu'à celle selon laquelle les souhaits du soi « antérieur » sont à préserver à tout prix (e.g. Dworkin 1986).

17 Aux fins du présent article, nous nous concentrons sur le fait suivant : le moment où une personne autrefois encouragée à réviser et à mettre à jour ses documents juridiques est découragée de le faire constitue un point tournant fondé sur des postulats cogninormatifs et cisnormatifs qui dépendent d'une perspective non intersectionnelle du soi. Tout en reconnaissant que, sur le plan juridique, ce point tournant repose sur la capacité de la personne à prendre des décisions précises, dans le cas des personnes vivant avec une démence, leur capacité n'est pas régulièrement évaluée en raison des biais cités plus haut. Nous comprenons qu'enlever l'agentivité décisionnelle de certaines personnes vise à protéger celles en situation de vulnérabilité et que, par conséquent, cela peut être justifiable, voire bénéfique, dans certaines circonstances. Cela étant dit, notre objectif est d'interroger la délégitimation et l'invalidation omniprésentes des capacités décisionnelles des personnes vivant avec une démence une fois jugées juridiquement incompétentes ${ }^{14}$. Nous reconnaissons que le fait de dénoncer les postulats cisnormatifs et cogninormatifs qui sous-tendent les interprétations juridiques de la compétence et de promouvoir l'agentivité et la capacité décisionnelle des personnes vivant avec une démence évoque des débats potentiellement contentieux.

18 Afin de mieux illustrer les postulats cisnormatifs et cogninormatifs susmentionnés et leurs impacts sur les expériences des PTVAD relatives à ces lois, nous présentons ici une étude de cas hypothétique impliquant l'auteur principal et l'auteure principale du présent article. Quoique cette étude de cas soit située dans un avenir hypothétique, elle est ancrée dans la réalité courante, c'est-à-dire le fait qu'Alexandre est un homme trans et que Marjorie est sa partenaire cis. L'étude de cas puise aussi dans les quelques cas anecdotiques de PTVAD qui expriment une "confusion" ou une "détransition » de genre comme celles discutées dans les écrits présentés dans la deuxième section. Imaginez qu'Alexandre a maintenant 80 ans et vit avec une démence depuis cinq ans. Bien qu'il soit encore «fonctionnel » dans de nombreuses sphères de sa vie, il vit tous les jours plusieurs périodes de confusion. Depuis quelques mois, les jours où il oublie qu'il a fait sa transition dans sa vingtaine deviennent de plus en plus nombreux. À ces moments, il réfère à lui-même en tant que femme, utilise des pronoms féminins pour parler de lui-même et insiste qu'on le maquille et lui donne des vêtements de femme. Avant son diagnostic de démence, Alexandre avait décidé, avec un soutien juridique, de préparer des documents détaillés de fin de vie. Il avait choisi Marjorie comme décisionnaire autorisée à la fois pour les décisions administratives/financières et pour 
ses soins personnels. Suivant un modèle similaire à celui proposé par le National Resource Center on LGBT Aging (2014), il a indiqué que, dans toutes les circonstances, il voulait qu'on utilise à son égard des pronoms masculins, qu'on maintienne son expression de genre masculine et qu'on l'enterre et se souvienne de lui comme homme. Il a précisé que le retour à un prénom de femme, ainsi qu'à une apparence et à des pronoms féminins, constituerait des violences cisgenristes. Les jours où Alexandre semble « confus » à propos de son identité de genre et que Marjorie lui rappelle qu'il est un homme trans, qu'il a fait sa transition il y a presque cinquante ans et qu'il a toujours voulu être traité comme un homme, Alexandre devient agressif, triste, déprimé et continue de demander des vêtements de femme. Tout le monde autour de lui est troublé, dont le personnel de la résidence et l'ensemble de son réseau de soutien. Personne ne sait s'il faut interagir avec lui comme femme ou s'il faut se conformer à l'identité dans laquelle il a vécu pendant plusieurs décennies, et donc imposer une continuité biographique. Que faire de son apparence externe, par exemple, le rasage, le maquillage et les vêtements? Quels pronoms utiliser? Faudrait-il continuer d'injecter sa dose hebdomadaire de testostérone afin de maintenir son équilibre hormonal masculin? Faudrait-il évaluer quotidiennement la situation et respecter l'identité de genre réclamée selon la journée ? Serait-il préférable de se conformer à la nouvelle identité féminine réclamée et, le cas échéant, que faire les jours où il se sent comme un homme et veut qu'on le traite ainsi ? Quel prénom et quelle identité utiliser lors de ses obsèques? D'une part, accepter d'utiliser un prénom de femme et des pronoms féminins les jours où Alexandre réclame une identité féminine pourrait sembler la solution la plus respectueuse dans le contexte de ses préférences du moment. D'autre part, cette approche peut être perçue comme une forme de violence dans le contexte des souhaits exprimés dans ses documents juridiques. Marjorie aussi se sent coincée entre deux possibilités : faire violence à Alexandre et à son identité masculine trans en refusant les volontés exprimées dans ses documents (juridiques) de fin de vie ou, inversement, faire violence à ses besoins et à ses souhaits actuellement variables en imposant une continuité biographique articulée par Alexandre dans sa vie prédémence.

Nous soutenons que la notion de continuité biographique mobilisée par certaines stratégies d'intervention auprès de personnes vivant avec une démence, qu'elles soient trans ou cis, est ancrée dans la cisnormativité et la cogninormativité. Selon des postulats cisnormatifs, il est présumé que les individus adhèrent à une identité de genre stable tout au cours de la vie, de l'enfance jusqu'à la vieillesse. La perturbation de cette stabilité, que ce soit en raison d'une transition ou d'une "détransition ", va à l'encontre de la norme selon laquelle la continuité de l'identité et de l'expression de genre est à conserver tout au long du parcours de vie. De telles hypothèses cisnormatives peuvent aussi augmenter les chances d'une personne à être jugée juridiquement " incompétente », car une personne dont l'identité/expression de genre est «instable » risque, d'un point de vue cisnormatif et cogniticiste, d'être perçue comme étant confuse. Même si la fluidité ou l'instabilité de genre ne mène pas directement au diagnostic de démence ou à un jugement d'« incompétence » juridique, le personnel de soins et les proches utilisent souvent ces indices pour initier des diagnostics ou pour évaluer la capacité juridique. En d'autres mots, moins l'identité de genre d'une personne est stable, plus son risque d'être perçue comme étant «folle » ( « crazy»), « aliénée » (« mad ») ou « démente » est élevé (Sandberg 2018). À partir de ce point de vue, l'instabilité, la fluidité et la non binarité de genre constituent des indices 
supplémentaires qu'une personne est peut-être en train de "perdre la tête". Paradoxalement, ces postulats relatifs à la stabilité se trouvent aussi au sein des discours transnormatifs ${ }^{15}$ reproduits par certaines institutions sociales, politiques, médicales et juridiques, ainsi que par certaines personnes trans elles-mêmes, qui proposent une version similaire de continuité biographique par le biais du discours d'être «né-es dans le mauvais corps » (Serano 2007 ; Bettcher 2014). Selon ces discours, les personnes trans ne sont pas comprises comme vivant une discontinuité d'identité de genre, mais comme des individus qui vivaient toujours, intérieurement, leur genre d'auto-identification.

Tout comme les hypothèses cisnormatives, les postulats cogninormatifs reposent également sur la continuité biographique, cette fois-ci axée sur l'idée qu'une personne aux capacités cognitives normatives est mieux placée qu'une personne en situation de handicap cognitif pour prendre des décisions sur ses traitements, ses soins, son logement et son genre, pour ne mentionner que celles-ci. La préparation des documents (juridiques) de fin de vie est fondée sur un cogniticisme puisque ces postulats cogninormatifs ont été intériorisés par la plupart des personnes, qu'elles soient trans ou cis. Comme l'observe Reynolds (2016:61), « l'expérience d'un corps sans handicap ne fournit pas automatiquement une perspective privilégiée sur l'expérience du handicap; au contraire, elle mène souvent à une mauvaise interprétation, un mauvais jugement et une mauvaise représentation de cette expérience ». L'incapacité d'imaginer que les personnes vivant avec un handicap cognitif possèdent toujours la capacité de prendre des décisions et d'exprimer leurs souhaits contribue à la dévaluation des personnes vivant avec une démence, perçues comme étant des personnes non participatives dont les contributions sont vides de sens et cela renforce les biais présents dans la planification préalable des soins, particulièrement l'attribution d'une plus grande valeur aux capacités cognitives qu'aux dimensions affectives et expérientielles (de Boer et al. 2010; Werner et Doron 2017 ; Young et al. 2019). En somme, les différences cognitives produites par la démence rendent souvent les personnes vivant avec une démence indignes d'être prises en considération par la loi et peuvent mener au refus des droits fondamentaux de la personne (Bartlett et O'Connor 2010; Bartlett 2014; Cahill 2018; Thomas et Milligan 2018; Shakespeare, Zeilig et Mittler 2019). Une puissante illustration de la dévalorisation des personnes vivant avec une démence occasionnée par la cogninormativité se trouve dans cet exemple fourni par une travailleuse sociale interviewée par Werner et Doron (2017: 1209) :

J'ai demandé à un juge s'il accepterait d'inviter la personne vivant avec une démence [à la cour] avant de prendre sa décision sur la tutelle. Il a répondu avec véhémence : Hors de question! Il ne comprenait même pas ce que je lui demandais [...] Il a dit: C'est une voie à sens unique; une fois qu'il/elle reçoit un diagnostic d'Alzheimer il est impossible pour lui [sic] de prendre des décisions, alors pourquoi l'inviter à se présenter en cour? Il est parfaitement clair qu'il faut nommer une tutrice ou un tuteur.

Le cisgenrisme et le cogniticisme constituent des systèmes d'oppression qui fonctionnent à de multiples niveaux: sociaux, politiques, médicaux, juridiques et épistémologiques. Sur le plan épistémologique, la discrimination créée par l'oppression et la violence contre les personnes trans ou celles vivant avec des handicaps cognitifs les dévalorise comme sujets connaissant. Miranda Fricker a forgé la notion d'« injustice épistémique ", un concept selon lequel "un mal est commis envers un individu 
spécifiquement dans sa capacité comme sujet connaissant». Fricker distingue deux types d'injustice épistémique: «l'injustice testimoniale» et "l'injustice herméneutique » :

L'injustice testimoniale se produit lorsque les préjugés d'un individu font qu'il accorde une crédibilité réduite aux paroles d'une personne locutrice; l'injustice herméneutique se produit à un stade antérieur, lorsqu'une lacune dans les ressources interprétatives collectives crée un désavantage injuste pour la personne qui tente de faire sens de ses expériences sociales. Un exemple de la première serait que les autorités policières ne vous croient pas parce que vous être une personne noire ; un exemple de la deuxième serait que vous souffrez de harcèlement sexuel au sein d'une culture qui ne possède pas encore ce concept critique. (Fricker 2007 : 1)

Dans un article publié avec Katharine Jenkins, Fricker approfondit sa notion de "marginalisation herméneutique", vue comme condition préalable à l'injustice herméneutique (Fricker et Jenkins 2017: 268). Elles soutiennent que "l'injustice herméneutique est l'injustice d'une tentative contrecarrée pour rendre intelligible une expérience sociale significative... tandis que la marginalisation herméneutique constitue un facteur causal significatif de cet échec. Une personne compte comme herméneutiquement marginalisée dans la mesure où elle fait partie d'un groupe qui contribue insuffisamment à la mise en commun de concepts et de significations sociales » (Fricker et Jenkins $2017: 268$ ).

On pourrait dire, suivant les travaux de Crichton, Carel et Kidd (2017), Young et al. (2019) et d'autres, que les personnes vivant avec une démence souffrent des deux formes d'injustice épistémique, ainsi que de formes de marginalisation herméneutique. D'une part, sur la base de leur diagnostic de démence, ces personnes vivent une injustice testimoniale parce qu'elles perdent leur crédibilité comme locutrices. D'autre part, les personnes vivant avec une démence souffrent d'injustice herméneutique parce qu'elles sont désavantagées par le manque d'outils conceptuels nécessaires pour rendre leurs expériences vécues compréhensibles à la fois pour elles-mêmes et pour les autres. En effet, étant donné que les discours dominants sur la démence sont marqués par la perte, le déclin, l'irrationalité et l'incapacité (Bartlett et O'Connor 2010; Bartlett 2014; Boyle 2014; Aubrecht et Keefe 2016; Ward et Price 2016 ; Sandberg 2018), il devient difficile pour les personnes vivant avec une démence de percevoir leur condition autrement. Il peut être difficile, par exemple, de percevoir leur oppression à travers le cogniticisme. De plus, comme l'expliquent Fricker et Jenkins (2017), l'injustice herméneutique va de pair avec la marginalisation herméneutique; c'est justement parce que les personnes vivant avec une démence sont herméneutiquement marginalisées et ne sont pas perçues comme locutrices légitimes qu'elles ne peuvent pas faire valoir leurs perspectives sur la démence, ce qui conduit à une expérience d'injustice herméneutique. Non seulement les trois formes d'injustice épistémique créent un cercle vicieux où chacune alimente les autres, mais, simultanément, elles façonnent et sont façonnées par le cogniticisme. Effectivement, la délégitimation sur le plan épistémique est fondée sur le cogniticisme, alors que le fait que les personnes vivant avec une démence demeurent « herméneutiquement marginalisées » alimente le système cogniticiste parce que les voix de ces personnes sont rarement écoutées ou perçues comme étant crédibles. Par conséquent, le système cogniticiste demeure incontesté. Pour en revenir à notre étude de cas hypothétique, la décision d'Alexandre, en préparant ses documents (juridiques) de fin de vie, de favoriser la stabilité de l'identité de genre par l'usage continu de son prénom, de pronoms masculins et d'une 
apparence masculine, sans égard aux souhaits de son soi futur, accorde une plus grande autorité à son esprit cognitivement "sain » qu'à son " esprit dément » et prend racine dans diverses formes d'injustice épistémique qui découlent de la cisnormativité et de la cogninormativité, et ce, tout en les alimentant.

Selon notre approche intersectionnelle, nous terminons cette section en puisant dans le champ des études critiques sur la race (Crenshaw 1991; Hill Collins 2000 ; Medina 2012 ; 2017), particulièrement la notion des " épistémologies de l'ignorance ", très pertinente au regard des réalités des personnes vivant avec une démence. Medina $(2012$; 2017) décrit la manière dont les formes d'injustice épistémique peuvent mener à une "mort épistémique » qui survient lorsque «les capacités épistémiques d'un sujet ne sont pas reconnues" (Medina, 2017: 254). Ceci est dû, en partie, aux "épistémologies de l'ignorance ", définies comme des processus actifs permettant aux groupes dominants, c'est-à-dire les personnes blanches dans le cas de Medina et les personnes aux capacités cognitives normatives dans le cas du présent article, d'ignorer «les faits, les expériences et les significations sociaux qui, en raison de l'oppression raciale [à laquelle nous ajoutons l'oppression cogniticiste], deviennent invisibles, inaudibles ou simplement inintelligibles dans certains contextes sociaux et pour certaines perspectives qui se protègent de faire face à leur participation dans l'oppression raciale [et cogniticiste] à l'aide d'un bouclier d'ignorance active » (Medina 2017 : 247). Comme le montrent les exemples plus haut, c'est précisément ce qui se passe dans le cas des personnes vivant avec une démence. Leurs voix, leurs préoccupations, leurs perspectives et leurs réalités sont activement oubliées par les individus qui les entourent, et cette injustice épistémique est exercée et reproduite par les notions cogniticistes d'« incapacité ». Dans la dernière section, nous explorons les possibilités pour aider les personnes vivant avec une démence à exercer leur agentivité.

\section{Les personnes trans vivant avec une démence parlent : pouvons-nous écouter ${ }^{16}$ ?}

Ayant exploré les manières dont les postulats cisnormatifs et cogninormatifs soustendent la planification et les documents (juridiques) de fin de vie des PTVAD, comment lutter contre les injustices épistémiques découlant de ces postulats afin de permettre aux PTVAD de parler non seulement avant de développer leur démence, mais aussi pendant qu'elles sont démentes? Et lorsqu'elles parlent, comment s'assurer qu'elles sont entendues? S'inspirant du concept de résistance épistémique de Medina (2017) et de la notion d'" approche trans-affirmative fluide » auprès des PTVAD de Baril et Silverman (2019), nous proposons des réflexions fondatrices visant à alimenter une reconceptualisation de la capacité et du consentement des PTVAD en fin de vie. Comme mentionné plus haut, l'intention de cet article n'est pas de fournir des solutions définitives à la modification potentielle des lois sur la capacité et des documents (juridiques) de fin de vie, mais d'amorcer un dialogue sur les meilleures stratégies pour soutenir les PTVAD et pour donner voix à leurs volontés.

Nous nous inspirons de Medina (2017), dont le concept d'épistémologie de la résistance reconnaît et donne voix aux personnes systématiquement réduites au silence par l'oppression et la marginalisation. Medina explique que «la résistance à l'oppression exige la création de frictions épistémiques sur le terrain, c'est-à-dire, par les pratiques qui exercent une pression et qui troublent dans l'objectif de faire cesser ou de 
perturber les dynamiques oppressives » (254). Une telle résistance, sous forme d'« actes insurrectionnels épistémiques » (256), peut être réalisée à la fois sur le plan personnel et structurel comme réponse à la violence quotidienne vécue par les groupes marginalisés. Il n'est pas nécessaire que ces actes soient de grands gestes; en fait, Medina explique que «les micro-pratiques de résistance méritent d'être exercées de manière concertée et continue parce que, tout comme un seul acte de micro-agression peut ne pas avoir un grand impact négatif, mais que, collectivement, les microagressions aident à maintenir une culture d'intimidation et de violence épistémique, les micro-activités de résistance [...] peuvent aider à miner, à affaiblir et finalement à détruire cette culture » $(2017: 258)$. Une notion similaire de résistance se trouve dans l'explication du concept de «cripistemologie » de Kim Hall, qui « considère en quoi les différences cognitives peuvent constituer des ressources épistémiques pour développer des savoirs résistants et transformateurs » $(2017: 165)$. Comme Medina, Hall fait appel aux pratiques de résistance qu'il est possible d'interpréter, dans le cas des personnes vivant avec une démence, comme la reconnaissance de formes non cogninormatives du savoir, notamment les désirs exprimés par les gestes, les actions non verbales ou les émotions.

27 Avant de conceptualiser ce à quoi pourrait ressembler ces micro-pratiques de résistance chez les PTVAD, il est important de nommer certains des éléments fondamentaux qui devraient sous-tendre ces pratiques et ces actions. Toute approche visant à lutter contre l'injustice épistémique et à donner voix aux PTVAD, que ce soit par le biais de grandes actions, comme la redéfinition de la capacité juridique, ou de micro-actions, comme une réplique à un regard méprisant, se doit d'être intersectionnelle. Une approche intersectionnelle aux enjeux uniques auxquels les PTVAD sont confrontées reconnaît que les diverses dimensions de l'identité, dont l'âge, le handicap cognitif et la transitude (sans oublier les autres facettes possibles, comme la race, selon l'individu) et leurs systèmes d'oppression connexes, dont l'âgisme, le cogniticisme et le cisgenrisme, s'entrecroisent pour mener à des formes de violences imbriquées. Par exemple, historiquement, les personnes handicapées de tous les âges ont été pathologisées et leurs voix réduites au silence en ce qui concerne leurs décisions de santé, de logement, de sexualité et ainsi de suite (Clare 2009). Pour les personnes âgées handicapées, cette violence capacitiste s'entrecroise avec l'âgisme, ce qui les rend encore plus invisibles et marginalisées, car les personnes âgées voient souvent leurs droits refusés et sont traitées comme sujets incompétents et non productifs (Bartlett 2014 ; Boyle 2014 ; Thomas et Milligan 2018 ; Shakespeare, Zeilig et Mittler 2019). L'ajout de la transitude à ce portrait peut mener, comme mentionné plus haut, au renforcement de l'idée que les personnes trans âgées vivant avec un handicap cognitif sont «folles» en raison d'une non conformité de genre. Dans un tel cas, la transformation ou la perturbation de l'identité de genre constituerait une "preuve » supplémentaire que l'agentivité devrait être refusée.

En plus d'offrir une critique des formes imbriquées d'oppression, une approche intersectionnelle peut également mener à trouver des solutions imbriquées. Dans leur typologie de réponses à la "détransition " potentielle de PTVAD, Baril et Silverman (2019) articulent une « approche trans-affirmative fluide » qui se veut intersectionnelle grâce à l'inclusion de l'âge, du handicap et de la transitude. En nous inspirant de cette approche, nous postulons que toute reconceptualisation de la compétence juridique ou de la planification et des documents (juridiques) de fin de vie pour les PTVAD, ainsi que toute action visant à lutter contre l'injustice épistémique et à améliorer leur qualité de 
vie, se doit d'être trans-affirmative et positive envers les réalités crip et celles des personnes âgées. Cela signifie que les PTVAD méritent de voir leur agentivité reconnue pour exprimer leur genre actuel, sans égard à sa conformité ou non avec une continuité biographique, ainsi que d'être entendues. Accorder aux PTVAD une agentivité qui leur permet d'exprimer leur genre choisi est en continuité avec une perception crip-positive de la démence, selon laquelle un handicap cognitif n'est pas compris comme menant à la perte de son identité individuelle ou de son agentivité. Une approche crip-positive valorise une variété de moyens d'expression de souhaits qui ne sont pas nécessairement basés sur les concepts cogninormatifs de la "rationalité ». Dans ce sens, une PTVAD serait en mesure d'exprimer son genre choisi par les gestes, le langage $\mathrm{du}$ corps, les vêtements, le maquillage et d'autres formes d'expression corporelles. L'agentivité permettant d'exprimer son genre de manière non cogninormative ne devrait non plus être refusée en cas d'âge avancé. Une approche positive envers l'âge encourage l'auto-détermination pour les individus de tout âge et vise à lutter contre l'invisibilisation et l'infantilisation souvent imposées aux personnes âgées et qui peuvent contribuer, dans le cas de personnes trans âgées, à contrôler leur identité et leur expression de genre.

Comment mettre en pratique ces principes? Comment exercer des pratiques de résistance qui favorisent l'agentivité des PTVAD? Suivant l'exemple de Joffe et Montigny (2014), Diller (2016) et d'autres juristes qui demandent une réévaluation de la capacité et de la prise de décision chez les personnes âgées et handicapées au Canada, ainsi que celui de Bach et Kerzner, qui préconisent une nouvelle approche "pour définir une capacité décisionnelle allant au-delà des présomptions capacitistes des lois sur la capacité actuellement en vigueur au Canada» (2010: 23), nous souhaitons également voir une conceptualisation plus large de la capacité qui tient compte de la multiplicité de manières dont les personnes vivant avec un handicap cognitif pourraient exprimer leurs volontés. Bach et Kerzner expliquent qu'il est possible de soutenir le processus décisionnel des personnes handicapées et que ces soutiens pourraient «mener à la capacité d'exprimer son intention ou sa volonté à l'aide d'une forme d'expression pouvant être raisonnablement comprise comme porteuse de sens par une autre personne» (2010:22). De tels soutiens pourraient inclure celui d'un individu qui aide la personne à interpréter et à représenter ses communications, lui explique les options dans un langage clair, lui accorde le temps qu'il lui faut au cours de plusieurs rencontres et lui répète les informations au besoin (Bach et Kerzner 2010 ; Diller 2016). L'idée d'offrir un soutien aux personnes vivant avec une démence pour qu'elles puissent maintenir leur agentivité constitue une approche activement positive envers les identités crip et les personnes âgées, mais elle cadre aussi avec la longue histoire des études féministes et crip/sur le handicap qui abordent le concept d'autonomie à partir d'une optique anti-oppression en théorisant ses dimensions relationnelles et l'importance de l'interdépendance et de l'accessibilité (Kafer 2013; Hall 2017).

30 Partant de l'idée que les PTVAD méritent un meilleur soutien pour exprimer leurs volontés pendant qu'elles vivent avec la démence et non seulement avant, nous proposons que, dans l'expérience vécue des PTVAD, la planification (juridique) de fin de vie et ses documents connexes constituent un processus continu, fluide et dynamique. Malgré l'appel à l'attribution d'une plus grande importance au processus de planification de fin de vie qu'à son résultat (de Vries et al. 2019), le processus est encore souvent conceptualisé comme ayant lieu avant qu'une personne ne développe un 
handicap cognitif. Nous sommes d'avis que le processus devrait continuer après que la personne développe un handicap cognitif et se poursuivre jusqu'à sa mort, une période pouvant être comptée en mois ou en années au cours desquels les capacités cognitives de la personne varieront probablement. Bien que nous reconnaissions que cette approche n'est pas sans embûches potentielles et qu'elle représente des défis pour les systèmes juridiques et de soins, ainsi que pour les familles, nous croyons que solliciter et soutenir les volontés des PTVAD de manière continue générerait des améliorations. Le processus de solliciter et de soutenir activement l'agentivité des PTVAD peut prendre la forme de rencontres régulières où l'on encourage la personne à exprimer ses volontés sur une variété de sujets (pas seulement le genre) et fournit un soutien pour les formes non verbales d'expression, comme le papier et les crayons si la personne veut dessiner ou des fiches d'images si elle veut choisir des images. On pourrait créer des environnements sécuritaires où toutes les formes d'expression de genre sont valorisées, que ce soit à l'aide de macarons indiquant les différents pronoms ou d'une variété de vêtements, de maquillage et d'accessoires pouvant appuyer une diversité d'expressions de genre (Baril et Silverman 2019). Il n'est pas possible de créer de tels environnements sécuritaires sans changements structurels connexes, par exemple, les salles de bain neutres de genre dans les établissements de soins, la formation et la sensibilisation du personnel et des membres du réseau de soutien personnel et la sensibilisation du public relative à la démence et aux réalités trans afin d'en réduire la stigmatisation. Finalement, les PTVAD ayant déjà préparé des documents (juridiques) de fin de vie devraient avoir l'occasion de les réviser régulièrement, à l'aide de mesures de soutien au besoin, afin de fournir leur consentement ou leur accord continu. Ainsi, nous n'encourageons pas les personnes trans à renoncer à la planification et aux documents (juridiques) de fin de vie. Nous encourageons plutôt les PTVAD et une grande variété de personnes dans leur réseau d'entamer un processus de réflexion critique sur certains des principes, des valeurs et des postulats implicites ayant une incidence sur la planification et les documents (juridiques) de fin de vie, ainsi que les lois applicables.

31 L'objectif des mesures de soutien susmentionnées est d'obtenir un consentement ou un accord continu et d'assurer un processus de planification (juridique) de fin de vie véritablement fluide qui résiste au cisgenrisme, au cogniticisme et à l'âgisme. Pour ce faire, il faut non seulement repenser le concept de capacité d'une perspective non cogniticiste et non cisnormative, mais aussi repenser la notion de consentement, en s'éloignant de la fixité pour privilégier la fluidité. De fait, si les documents juridiques sont régulièrement repensés, réaffirmés et réitérés par les personnes vivant avec une démence, ces documents ne doivent pas être compris comme un résultat «final » ni comme le «dernier» mot d'une personne. Tout comme le consentement sexuel fut repensé par les féministes de manière à impliquer maintenant un consentement continu et affirmatif, nous croyons que la planification (juridique) de fin de vie devrait être offerte aux personnes vivant avec une démence, et à la population entière, à titre de processus continuellement renouvelé et affirmé.

32 Même si cet article est axé sur les PTVAD, nous espérons que les réflexions ici présentées serviront de fondements à une exploration plus approfondie de l'agentivité de personnes vivant avec un handicap cognitif ou celles jugées juridiquement « incompétentes » (Bach et Kerzner 2010 ; Reynolds 2016 ; Crichton, Carel et Kidd 2017). Nous espérons que les juristes et les autres individus concernés relèveront le défi d'interroger les postulats cogniticistes, cisgenristes et âgistes qui se trouvent au cœur 
des notions de la capacité et de la planification et des documents (juridiques) de fin de vie pour les communautés trans. De nouveaux discours juridiques émergents prônent le passage des «régimes de prise de décision autorisée [...] [à la] prise de décision soutenue qui respecte l'autonomie, la volonté et les préférences de la personne " (Cahill 2018: 160). De plus, certains travaux juridiques commencent à discuter de la nécessité d'approches intersectionnelles dans le domaine juridique afin de mieux comprendre l'expérience vécue d'individus qui font partie de multiples groupes marginalisés pour que la loi puisse les protéger adéquatement de l'oppression, au lieu de perpétuer les violences (Bilge et Roy 2010 ; Spade 2013; Clutterbuck 2015; Singer 2020). Nous encourageons fortement ces avancées, de la même manière que nous encourageons le militantisme croissant des personnes vivant avec une démence (Bartlett 2014) et leur inclusion dans les dialogues sur la réforme collaborative et intersectionnelle des lois touchant les communautés trans et les personnes handicapées. Le temps est venu de laisser parler les personnes (trans) vivant avec une démence. Le temps est venu d'écouter.

\section{BIBLIOGRAPHIE}

ADELMAN, Marcy. 2016. « Overcoming barriers to care for LGBT elders with Alzheimer's Generations » Journal of the American Society on Aging 40 (2) : 38-40.

ALMACK, Kathryn. 2019. " "I didn't come out to go back in the closet": Ageing and end-of-life care for older LGBT people ", in Older lesbian, gay, bisexual and trans people. Minding the knowledge gaps, KING, Andrew, ALMACK, Kathryn, SUEN, Yiu-Tung \& WESTWOOD, Sue (éds.). Oxon : Routledge, 158-171.

Alzheimer's Australia. 2014. Dementia, transgender and intersex people: Do service providers really know what their needs are? Melbourne : Alzheimer Society.

ANSARA, Y. Gavriel. 2015. « Challenging cisgenderism in the ageing and aged care sector: Meeting the needs of older people of trans and/or non-binary experience » Australasian Journal on Ageing $34: 14-18$.

AUBRECHT, Katie \& KEEFE, Janice. 2016. « The becoming subject of dementia » Review of Disability Studies: An International Journal 12 (2 \& 3) : 1-19.

BACH, Michael \& KERZNER, Lana. 2010. A new paradigm for protecting autonomy and the right to legal capacity. advancing substantive equality for persons with disabilities through law, policy and practice. Ontario : The Law Commission of Ontario.

BAILEY, Louis. 2012. «Trans ageing. Thoughts on a life course approach in order to better understand trans lives » in Lesbian, gay, bisexual and transgender ageing. Biographical approaches for inclusive care and support, WARD, Richard, RIVERS, Ian \& SUTHERLAND, Mike (éds.). London : Jessica Kingsley Publishers, 51-66.

BARIL, Alexandre. 2009. «Transsexualité et privilèges masculins. Fiction ou réalité ?» in Diversité sexuelle et constructions de genre, CHAMBERLAND, Line, FRANK, Blye W. \& RISTOCK, Janice (éds.). Québec : Presses de l’Université du Québec, 263-295. 
BARIL, Alexandre. 2019. « An analysis of the underrepresentation of trans professors in Canadian universities » Chiasma: A Site for Thought $5:$ 90-128.

BARIL, Alexandre \& SILVERMAN, Marjorie. 2019. « Forgotten lives: Trans older adults living with dementia at the intersection of cisgenderism, ableism/cogniticism and ageism » Sexualities [En ligne], consulté le 5 août 2020. URL : https://doi.org/10.1177/1363460719876835.

BARRETT, Catherine, CRAMERI, Pauline, LATHAM, J. R., WHYTE, Carolyn \& LAMBOURNE, Sally. 2016. « Person-centered care and cultural safety. The perspective of lesbian, gay, and trans (LGT) people and their partners on living with dementia » in Lesbian, gay, bisexual, and trans individuals living with dementia. Concepts, practice and rights, WESTWOOD, Sue \& PRICE, Elizabeth (éds.). Abingdon : Routledge 97-109.

BARTLETT, Ruth. 2014. «Citizenship in action: The lived experiences of citizens with dementia who campaign for social change » Disability \& Society 29 (8) : 1291-1304.

BARTLETT, Ruth, \& O'CONNOR, Deborah. 2010. Broadening the dementia debate. Bristol: The Policy Press.

BAUER, Greta R. \& SCHEIM, Ayden I. 2015. Transgender people in Ontario, Canada: Statistics from the Trans PULSE Project to Inform Human Rights Policy. Londres, Ontario : University of Western Ontario, consulté le 5 août 2020. URL : http://transpulseproject.ca/wp-content/uploads/2015/06/TransPULSE-Statistics-Relevant-for-Human-Rights-Policy-June-2015.pdf

BAUER, Greta R., HAMMOND, Rebecca, TRAVERS, Robb, KAAY, Matthias, HOHENCADEL, Karin M. \& BOYCE, Michelle. 2009. " "I don't think this is theoretical; this is our lives": How erasure impacts health care for transgender people » The Journal of the Association of Nurses in AIDS Care 20 (5) : 348-361.

BETTCHER, Talia Mae. 2014. « Trapped in the wrong theory: Rethinking trans oppression and resistance » Signs 39 (2) : 383-406.

BILGE, Silma, \& ROY, Olivier. 2010. «Discrimination intersectionnelle : La naissance et le développement d'un concept et les paradoxes de sa mise en application en droit antidiscriminatoire » Canadian Journal of Law and Society 25 (1) : 51-74.

BOYLE, Geraldine. 2014. « Recognising the agency of people with dementia » Sociology 29 (7) : 1130-1144.

BUCHANAN, Allen. 1988. " Advance directives and the personal identity problem » Philosophy and Public Affairs 17 : 277-302.

BURY, Michael. 1982. "Chronic illness as biographical disruption » Sociology of Health \& Illness 4 (2) : 167-182.

CAHILL, Suzanne. 2018. Dementia and human rights. Bristol : Policy Press.

Canadian Association for Community Living. 2019. "What is legal capacity? » consulté le 5 août 2020. URL : https://cacl.ca/2017/11/15/what-is-legal-capacity/.

CARTWRIGHT, Colleen. 2011. Respect my decisions: It's my right! A guide to advance end-of-life care planning for gay, lesbian, bisexual, transgender and intersex people. Lismore : ASLaRC Aged Services Unit of Southern Cross University.

CARTWRIGHT, Colleen, WHITE, Ben, WILLMOTT, Lindy, PARKER, Malcom \& WILLIAMS, Gail. 2018. « Australian doctors' knowledge of and compliance with the law relating to end-of-life decisions: Implications for LGBTI patients »Culture, Health \& Sexuality 20 (8) : 845-857. 
CHAKRAVORTY SPIVAK, Gayatri. 1988. «Can the subaltern speak? » in Marxism and the interpretation of culture, NELSON, Cary \& GROSSBERG, Lawrence (éds.). Champaign : University of Illinois Press, 271-313.

CHAMBERLAND, Line, BEAUCHAMP, Julie, DUMAS, Jean \& KAMGAIN, Olivia. 2016. Aîné.e.s LGBT : favoriser le dialogue sur la préparation de leur avenir et de leur fin de vie, et la prise en charge communautaire. Montréal : Chaire de recherche sur l'homophobie, UQAM.

CLARE, Eli. 2009 [1999]. Exile \& pride : Disability, queerness and liberation. New York : South End Press.

CLUTTERBUCK, Alyssa. 2015. « Rethinking Baker: A critical race feminist theory of disability » Appeal: Review of Current Law and Law Reform 20 : 51-70.

Combahee River Collective. 1997. « A black feminist statement [1977] » in The second wave. A reader in feminist theory, NICHOLSON, Linda (éd.). New York : Routledge, 63-70.

COOK-DANIELS, Loree. 2006. «Trans aging » in Lesbian, gay, bisexual and transgender aging: Research and clinical perspectives, KIMMEL, Douglas, ROSE, Tara \& DAVID, Steven (éds.). Milwaukee :

Columbia University Press.

CRENSHAW, Kimberlé. 1991. « Mapping the margins: Intersectionality, identity politics, and violence against women of color » Stanford Law Review 43 (6) : 1241-1299.

CRICHTON, Paul, CAREL, Havi \& KIDD, Ian James. 2017. « Epistemic injustice in psychiatry » BJPsych Bulletin $41:$ 65-70.

DAVIS, John K. 2002. « The concept of precedent autonomy » Bioethics $16: 114-133$.

DAVIS, John K. 2004. « Precedent autonomy and subsequent consent » Ethical Theory and Moral Practice 7 : 267-291.

DE BOER, Marike E., HERTOGH, Cees M. P. M., DRÖES, Rose-Marie, JONKER, Cees \& EEFSTING, Jan A. 2010. « Advance directives in dementia: Issues of validity and effectiveness » International Psychogeriatrics 22 (2) : 201-208.

DEGRAZIA, David. 1999. «Advance directives, dementia and "the someone else problem" " Bioethics 13 : 373-391.

DE VRIES, Brian, GUTMAN, Gloria, HUMBLE, Áine, GAHAGAN, Jacqueline, CHAMBERLAND, Line, AUBERT, Patrick, FAST, Janet \& MOCK, Steven. 2019. « End-of-life preparations among LGBT older Canadian adults: The missing conversations » The International Journal of Aging and Human Development 88 (4) : 358-379.

DILLER, Rebekah. 2016. «Legal capacity for all: Including older persons in the shift from adult guardianship to supported decision-making » Fordham Urban Law Journal 43 (3) : 494-538.

DRESSER, Rebecca. 1995. « Dworkin on dementia » Hastings Center Report 25 : 32-8.

DWORKIN, Ronald. 1986. « Autonomy and the demented self » The Milbank Quarterly, 64 (Suppl. 2) : 4-16.

FABBRE, Vanessa D. 2015. « Gender transitions in later life: A queer perspective on successful aging » The Gerontologist 55 (1) : 144-153.

FINKENAUER, Sabine, SHERRATT, Jackson, MARLOW, Jean \& BRODLEY, Andrea. 2012. « When injustice gets old: A systemic review of trans aging » Journal of Gay \& Lesbian Social Services 24 (4) : 311-330. 
FLYNN, Eilionóir. 2018. « Legal capacity for people with dementia: A human rights approach » in Dementia and Human Rights, CAHILL, Suzanne (éd). Bristol : Bristol University Press, Policy Press, 157-174

FRICKER, Miranda. 2007. Epistemic Injustice: Power and the Ethics of Knowing. New York : Oxford University Press.

FRICKER, Miranda \& JENKINS, Katharine. 2017. « Epistemic injustice, ignorance and trans experiences » in Routledge Companion to Feminist Philosophy, GARRY, Ann, KHADER, Serene J. \& STONE, Alison (éds.). New York : Routledge, 268-278.

FURBERG, Elisabeth. 2012. «Advance directives and personal identity: What is the problem? » Journal of Medicine and Philosophy $37: 60-73$.

GORMAN, Rachel \& LEFRANÇOIS, Brenda A. 2017. « Mad studies » in Routledge International Handbook of Critical Mental Health, COHEN Bruce M. Z. (éd.). Londres : Routledge, 107-114.

HALL, Kim Q. 2017. «Queer epistemology and epistemic injustice » in The Routledge handbook of epistemic injustice, KIDD, Ian James, MEDINA, José \& POHLHAUS JR. Gaile (éds.). New York : Routledge, 158-166.

HALL, Margaret Isabel. 2015. « Dementia, decision-making, and the modern (adult) guardianship paradigm: Bentley v. Maplewood Seniors Care Society » Canadian Journal of Comparative and Contemporary Law 1 (1) : 293-316.

Health Law Institute. 2020. « Advance directives, end-of-life law \& policy in Canada » Dalhousie University. consulté le 5 août 2020. URL : Eol.law.dal.ca/?page_id=231

HÉBERT, William, CHAMBERLAND, Line \& ENRIQUEZ, Mickael Chacha. 2012. « Les aîné-es trans : une population émergente ayant des besoins spécifiques en soins de santé, en services sociaux et en soins liés au vieillissement » Frontières 25 (1) : 57-81.

HILL COLLINS, Patricia. 2000 [1990]. Black feminist thought: Knowledge, consciousness, and the politics of empowerment. New York Routledge.

HOGAN, Sally. 2017. « Diversity in sexuality combined with a diagnosis of dementia leads to poor health outcomes for LGBTIQ older persons » Australian Nursing \& Midwifery Journal 25 (5) : 24.

HUGHES, Mark. 2016. « Providing responsive services to LGB individuals with dementia » In Lesbian, gay, bisexual, and trans individuals living with dementia. Concepts, practice and rights, WESTWOOD, Sue \& PRICE, Elizabeth (éds.). Abingdon : Routledge, 83-96.

HUGHES, Mark \& CARTWRIGHT, Colleen. 2015. « Lesbian, gay, bisexual and transgender people's attitudes to end-of-life decision-making and advance care planning » Australasian Journal on Ageing 34 (2) : 39-43.

HULKO, Wendy. 2016. «LGBT individuals and dementia. An intersectional approach » in Lesbian, Gay, bisexual, and trans individuals living with dementia. Concepts, practice and rights, WESTWOOD, Sue \& PRICE, Elizabeth (éds.). Abingdon : Routledge, 35-50.

HUNTER, Chryssy, BISHOP, Jenny-Anne \& WESTWOOD, Sue. 2016. « The complexity of trans/ gender identities. Implications for dementia care » in Lesbian, gay, bisexual, and trans individuals living with dementia. Concepts, practice and rights, WESTWOOD, Sue \& PRICE, Elizabeth (éds.). Abingdon : Routledge, 124-137.

JAMES, J., BAUER, Greta, PECK, Ryan, BRENNAN, David \& NUSSBAUM, Nicole. 2018. Legal problems facing trans people in Ontario. TRANSforming JUSTICE Summary Report 1 (1). 
JAWORSKA, Agnieszka. 1999. « Respecting the margins of agency: Alzheimer's patients and the capacity to value » Philosophy and Public Affairs $28: 105-138$.

JOFFE, Kerri \& MONTIGNY, Edgar-Andre. 2014. Decisions, decisions: Promoting and protecting the rights of persons with disabilities who are subject to guardianship. Ontario : The Law Commission of Ontario.

JOHNSON, Austin H. 2016. « Transnormativity: A new concept and its validation through documentary film about transgender men » Sociological Inquiry 20 (10) : 1-27.

KAFER, Alison. 2013. Feminist, queer, crip. Bloomington : Indiana University Press.

KATTARI, Shanna K. \& HASCHE, Leslie. 2016. « Differences across age groups in transgender and gender non-conforming people's experiences of health care discrimination, harassment, and victimization » Journal of Aging and Health 28 (2) : 28-306.

KIA, Hannah. 2015. « Hypervisibility: Toward a conceptualization of LGBTQ aging » Sexuality Research and Social Policy 13 (1) : 46-57.

KING, Andrew. 2016. «Queer(y)ing dementia - Bringing queer theory and studies of dementia into dialogue " in Lesbian, gay, bisexual, and trans individuals living with dementia. Concepts, practice and rights, WESTWOOD, Sue \& PRICE, Elizabeth (éds.). Abingdon : Routledge, 51-64.

KING, Andrew, ALMACK, Kathryn, SUEN, Yiu-Tung \& WESTWOOD, Sue (éds.). 2019. Older lesbian, gay, bisexual and trans people. Minding the knowledge gaps. New York : Routledge.

KNAUER, Nancy J. 2016. « LGBT individuals living with dementia. rights and capacity issues in the United States » in Lesbian, gay, bisexual, and trans individuals living with dementia. Concepts, practice and rights, WESTWOOD, Sue \& PRICE, Elizabeth (éds.). Abingdon : Routledge, 173-189.

LATHAM, J. R. \& BARRETT, Catherine. 2015a. Trans health and ageing: An evidence-based guide to inclusive services. Melbourne, Australie : Australian Research Centre in Sex, Health and Society, La Trobe University.

LATHAM, J. R. \& BARRETT, Catherine. 2015b. Gender is just part of who I am: Stories from trans Australians: Exploring the experiences and needs of trans people for health and aged care services. Melbourne, Australie : Australian Research Centre in Sex, Health and Society, La Trobe University.

LEFRANÇOIS, Brenda A., MENZIES, Robert \& REAUME, Geoffrey (éds.). 2013. Mad matters: A critical reader in Canadian mad studies. Toronto : Canadian Scholars' Press.

MARSHALL, Jeanine, COOPER, Michael \& RUDNICK, Abraham. 2015. « Gender dysphoria and dementia: A case report » Journal of Gay \& Lesbian Mental Health 19 : 112-117.

MCFADDEN, Susan H., FRANKOWSKI, Scott \& WITTEN, Tarynn. 2017. « Anticipating the possibility of developing dementia: Perspectives of older transgender/intersex persons » Alzheimer Europe, consulté le 5 août 2020. URL : http://www.alzheimer-europe.org/content/download/ 24282/173003/file/P18.5\%20Mcfadden.pdf.

MCGOVERN, Justine. 2014. « The forgotten: Dementia and the aging LGBT community » Journal of Gerontological Social Work $57: 845-857$.

MCRUER, Robert. 2006. Crip theory: Cultural signs of queerness and disability. New York : New York University Press.

MEDINA, José. 2012. The epistemology of resistance: Gender and racial oppression, epistemic injustice, and resistant imaginations. New York : Oxford University Press. 
MEDINA, José. 2017. « Epistemic injustice and epistemologies of ignorance » in Routledge companion to philosophy of race, TAYLOR, Paul C., ALCOFF, Linda Martin \& ANDERSON, Luvell (éds.). New York : Routledge, 247-260.

MOODY, Janis. 2003. « Dementia and personhood: Implications for advance directives » Nursing older People 15 (4) : 18-21.

NAMASTE, Viviane K. 2000. Invisible lives: The erasure of transsexual and transgendered people. Chicago : The University of Chicago Press.

National End of Life Care Programme. 2012. The route to success in end of life care: Achieving quality for lesbian, gay, bisexual and transgender people [En ligne], consulté le 5 août 2020. URL : https:// www.england.nhs.uk/improvement-hub/wp-content/uploads/sites/44/2017/10/End-of-LifeCare-Route-to-Success-Achieving-Quality.pdf.

National Resource Center on LGBT Aging. 2014. Creating end-of-life documents for trans individuals: An advocate's guide [En ligne], consulté le 5 août 2020. URL : https://www.whitman-walker.org/ Guides\%20PDF/End\%20of\%20Life\%20Planning.pdf.

NEWMAN, Roger. 2016. « The needs and rights of LGBT carers of individuals with dementia: a personal journey » in Lesbian, gay, bisexual, and trans individuals living with dementia. Concepts, practice and rights, WESTWOOD, Sue \& PRICE, Elizabeth (éds.). Abingdon : Routledge, 190-202.

NEWMAN, Roger \& PRICE, Elizabeth. 2012. « Meeting the needs of LGBT people affected by dementia. The story of the LGBT dementia support group » in Lesbian, gay, bisexual and transgender ageing: Biographical approaches for inclusive care and support, WARD, Richard, SUTHERLAND, Mike \& RIVERS, Ian (éds.). Londres : Jessica Kingsley Publishers, 103-109.

Ontario Medical Association (OMA). 2014. Advance care planning: backgrounder. OMA's end-of-life care strategy [En ligne], consulté le 5 août 2020. URL : https://content.oma.org//wp-content/uploads/ advancedcareplanning.pdf.

PANG, Celeste, GUTMAN, Gloria \& DE VRIES, Brian. 2019. « Later life care planning and concerns of transgender older adults in Canada » International Journal of Aging \& Human Development 89 (1) : 39-56.

PARFIT, Derek. 1984. Reasons and persons. Oxford : Oxford University Press.

PEARCE, Ruth. 2019. « Trans temporalities and non-linear ageing » in Older lesbian, gay, bisexual and trans people. Minding the knowledge gaps, KING, Andrew, ALMACK, Kathryn, SUEN, Yiu-Tung \& WESTWOOD, Sue (éds.). Oxon : Routledge, 61-74.

PEEL, Elizabeth \& MCDAID, Sam. 2015. "Over the Rainbow" lesbian, gay, bisexual and trans people and dementia project: Summary report. Worcester : Institute of Health and Society.

PERSSON, Diane I. 2009. « Unique challenges of transgender aging: implications from the literature » Journal of Gerontological Social Work 52 : 633-646.

REYNOLDS, Joel Michael. 2016. « The ableism of quality of life judgments in disorders of consciousness: Who bears epistemic responsibility? » AJOB Neuroscience 7 (1) : 59-61.

SABAT, Steven \& HARRÉ, Rom. 1992. « The Construction and deconstruction of self in Alzheimer's disease » Ageing and Society 12 (4) : 443-461.

SANDBERG, Linn J. 2018. « Dementia and the gender trouble?: Theorising dementia, gendered subjectivity and embodiment » Journal of Aging Studies 45 : 25-31.

SERANO, Julia. 2007. Whipping girl. A transsexual woman on sexism and the scapegoating of femininity. Berkeley : Seal Press. 
SHAKESPEARE, Tom, ZEILIG, Hannah \& MITTLER, Peter. 2019. « Rights in mind: Thinking differently about dementia and disability » Dementia 18 (3) : 1075-1088.

SINGER, Samuel. 2020 forthcoming. « Trans justice, trans rights: A multi-instrumentalist legal toolkit » Canadian Journal of Law and Society.

SINGER, Samuel. 2019. " Trans competent lawyering » in LGBTQ2+ law: Practice issues and analysis, RADBORD, Joanna (éd.). Toronto : Emond Publishing, 159-186.

SIVERSKOG, Anna. 2015. « Ageing bodies that matter: Age, gender and embodiment in older transgender people's life stories » NORA-Nordic Journal of Feminist and Gender Research 23 (1) : 4-19.

SPADE, Dean. 2013. «Intersectional resistance and law reform » Signs: Journal of Women in Culture and Society 38 (4) : 1031-1055.

STEIN, Gary L. \& ALMACK, Kathryn. 2012. « Care near the end of life. The concerns, needs, and experiences of LGBT elders » in Lesbian, gay, bisexual and transgender ageing. Biographical approaches for inclusive care and support, WARD, Richard, RIVERS, Ian \& SUTHERLAND, Mike (éds.). London : Jessica Kingsley Publishers, 114-131.

THOMAS, Carol \& MILLIGAN, Christine. 2018. « Dementia, disability rights and disablism: Understanding the social position of people living with dementia » Disability \& Society 33 (1) : $115-131$.

TOZE, Michael. 2018. « Developing a critical trans gerontology » British Journal of Sociology, 1-20.

VERMA, Sarita \& SILBERFELD, Michel. 1997. « Approaches to capacity and competency: The Canadian view » International Journal of Law and Psychiatry 20 (1) : 35-46.

WARD, Richard, RIVERS, Ian \& SUTHERLAND, Mike (éds.). 2012. Lesbian, gay, bisexual and transgender ageing. Biographical approaches for inclusive care and support, London : Jessica Kingsley Publishers.

WARD, Richard \& PRICE, Elizabeth. 2016. « Reconceptualising dementia. Toward a politics of senility » in Lesbian, gay, bisexual, and trans individuals living with dementia. Concepts, practice and rights, WESTWOOD, Sue \& PRICE, Elizabeth (éds.). Abingdon : Routledge, 65-77.

WERNER, Perla \& DORON Israel (Issi). 2017. « Alzheimer's disease and the law: Positive and negative consequences of structural stigma and labeling in the legal system » Aging and Mental Health 21 (11) : 1206-1213.

WESTWOOD, Sue. 2016. « Gender, sexuality, gender identity and dementia. (In)equality issues » in Lesbian, gay, bisexual, and trans individuals living with dementia. Concepts, practice and rights, WESTWOOD, Sue \& PRICE, Elizabeth (éds.). Abingdon : Routledge, 21-34.

WESTWOOD, Sue \& PRICE, Elizabeth (éds.). 2016. Lesbian, gay, bisexual, and trans individuals living with dementia. Concepts, practice and rights. Abingdon : Routledge.

WILSON, Kimberley, KORTES-MILLER, Katherine \& STINCHCOMBE, Arne. 2018. « Staying out of the closet: LGBT older adults' hopes and fears in considering end-of-life » Canadian Journal on Aging 37 (1) : 22-31.

WITHALL, Liz. 2014. Dementia, transgender and intersex people: Do service providers really know what their needs are? Australia : Alzheimer's Australia.

WITTEN, Tarynn M. 2014. « End of life, chronic illness, and trans-identities » Journal of Social Work in End-of-Life \& Palliative Care 10 (1): 34-58.

WITTEN, Tarynn M. 2016. « Trans people anticipating dementia care. Findings from the Transgender MetLife Survey » in Lesbian, gay, bisexual, and trans* individuals living with dementia. 
Concepts, practice and rights, WESTWOOD, Sue \& PRICE, Elizabeth (éds.). Abingdon : Routledge, 111-123.

WITTEN, Tarynn M. 2017. « Health and well-being of transgender elders » Annual Review of Gerontology and Geriatrics 37 (1) : 27-42.

WITTEN, Tarynn M. \& EYLER, A. Evan. 2012. Gay, lesbian, bisexual and transgender aging. Challenges in research, practice and policy. Baltimore : Johns Hopkins University Press.

YOUNG, Jessica A., LIND, Christopher, ORANGE, J. B. \& SAVUNDRANAYAGAM, Marie-Y. 2019. « Expanding Current understandings of epistemic injustice and dementia: Learning from stigma theory » Journal of Aging Studies $48: 76-84$.

\section{NOTES}

1. Traduit de l'anglais par Catriona LeBlanc. Sauf indication contraire, les citations dans le présent texte représentent des traductions libres de l'anglais. Les auteures et auteur souhaitent remercier la Canadian Journal of Law \& Society/Revue Canadienne Droit et Société pour sa permission de traduire et de reproduire cet article. Source de la publication originale: Baril, A., M. Silverman, M.-C. Gauthier et M. Lévesque (2020). « Forgotten Wishes: End-of-life documents for trans people with dementia at the margins of legal change ", Canadian Journal of Law \& Society/ Revue Canadienne Droit et Société, Volume 35, no. 2, p. 367-390. doi:10.1017/cls.2020.13

2. Le terme "trans" englobe une diversité d'identités de genre, dont celles transsexuelles, transgenres, non binaires, fluides de genre et bispirituelles (Baril, 2009).

3. Le terme "démence" comprend de nombreuses conditions pouvant occasionner des changements sur les plans de la mémoire, de la communication et de la cognition, parmi d'autres. La maladie d'Alzheimer et la démence vasculaire en sont des exemples.

4. L'Association canadienne pour l'intégration communautaire (Canadian Association for Community Living 2019) définit la capacité juridique ainsi : « [1]e terme reconnaît deux choses : la capacité d'avoir des droits et la capacité d'agir en vertu de ces droits. En pratique, la capacité juridique veille à ce qu'une personne soit reconnue devant la loi et puisse prendre des décisions concernant sa propre vie, exercer ses droits, avoir accès au système civil et juridique, conclure des contrats et parler en son propre nom.» [N. de T.: Traduction tirée du site Web de l'Association canadienne pour l'intégration communautaire.] Au Canada, la capacité juridique est présumée pour tout individu, exception faite des personnes mineures. Comme cette présomption est réfutable, il peut être décidé qu'une personne vivant avec une démence ne possède pas la capacité de prendre de décisions relatives aux soins de santé.

5. Nous utilisons l'expression « document (juridique) de fin de vie » et " planification (juridique) de fin de vie » avec le mot «juridique » entre parenthèses afin de souligner le fait que certains types de document et de planification comprennent à la fois des éléments juridiquement contraignants et d'autres qui ne le sont pas. En utilisant les parenthèses, nous cherchons à indiquer clairement l'inclusion des éléments à la fois juridiquement contraignants et non contraignants des documents et de la planification de fin de vie.

6. Le préfixe «cis» est l'opposé de «trans": une personne cisgenre/cissexuelle est donc une personne non transgenre/transsexuelle (Serano 2007). Bien que le cisgenrisme puisse être considéré comme un synonyme de la transphobie, ce premier terme présente l'avantage d'éviter la pathologisation individuelle associée à la notion de " phobie ». Le mot « cisgenrisme » souligne plutôt les formes de violence structurelles vécues par les communautés trans (Ansara 2015 ; Baril 2019).

7. S'inspirant des études critiques sur le handicap (Clare, 2009), des études crip (McRuer 2006 ; Kafer 2013) et des études sur la folie (mad studies) (LeFrançois, Menzies et Reaume 2013 ; Gorman 
et LeFrançois 2017) et leur problématisation des systèmes d'oppression des personnes handicapées (capacitisme) et des personnes folles ( $\mathrm{mad}$ ) et aliénées (insane) (deux termes réclamés et resignifiés par les mouvements militants), le terme "cogniticisme » est «compris comme un système d'oppression qui discrimine contre les personnes vivant avec un handicap cognitif/mental [...] à plusieurs niveaux, dont politiques, sociaux, médicaux, juridiques, économiques et normatifs" (Baril et Silverman 2019: 12). Nous utilisons ici trois termes, capacitisme/sanisme/cogniticisme, pour souligner le fait que le cogniticisme, quoiqu'il soit un excellent terme pour aborder l'oppression vécue par les personnes vivant avec des handicaps cognitifs, peut être considéré comme un synonyme du sanisme et représente une forme particulière de capacitisme.

8. Nous faisons appel à la définition d'intersectionnalité fournie par Crenshaw (1991) dans la tradition des études féministes noires comme dans les réflexions du Combahee River Collective (1997) et de Patricia Hill Collins (2000), parmi d'autres, qui soutiennent que les dimensions de l'identité comme le sexe, la race, la classe, l'âge, l'(in)capacité et leurs systèmes d'oppression connexes sont imbriqués.

9. Le néologisme « cisnormativité », inspiré par des expressions comme « hétéronormativité » et se basant sur des termes comme "cisgenrisme » et "cissexisme » (Serano 2007), a émergé simultanément en français et en anglais dans les travaux de Baril (2009) et de Bauer et al. (2009). Suivant Baril (2019), qui a affiné le concept dans ses travaux subséquents, nous comprenons la cisnormativité comme composante normative du système cisgenriste.

10. King $(2016,59)$ a forgé les termes « cogninormatif/cogninormativité » afin de problématiser les normes touchant les capacités cognitives dans les sociétés où les personnes vivant avec des handicaps cognitifs, dont les personnes vivant avec une démence, sont marginalisées.

11. Nous offrons la définition de théorie crip fournie par Baril et Silverman (2019:12): «La théorie crip [crip theory], ainsi nommée par Robert McRuer (2006), de manière similaire à la réclamation de l'injure "queer " par la théorie queer, resignifie l'injure "crippled " ( estropié ») et représente une perspective anti-assimilationniste au sein des études sur le handicap. Les perspectives anti-capacitistes et crip nous permettent d'adopter un point de vue critique sur le modèle biomédical de la démence qui l'associe au déclin, à la perte d'identité individuelle et au manque d'agentivité. »

12. Par exemple, une personne peut être jugée juridiquement compétente pour prendre une décision de santé mineure tout en étant jugée incompétente pour prendre une décision majeure relative à sa santé qui nécessiterait un raisonnement plus élaboré et une plus grande capacité décisionnelle.

13. Pour une discussion plus approfondie de ces débats, nous recommandons les sources suivantes : Parfit (1984) ; Dworkin (1986); Buchanan (1988); Dresser (1995); Verma et Silberfeld (1997) ; De Grazia (1999) ; Jaworska (1999); Davis (2002 ; 2004); Moody (2003); de Boer et al. (2010) ; Furberg (2012) ; Boyle (2014) ; Hall (2015) ; Cahill (2018) ; Flynn (2018).

14. Même si nous aimerions aborder les importants désavantages associés à l'attribution de la capacité juridique et de prise de décision aux individus vivant avec des handicaps cognitifs sévères qui peuvent ne pas comprendre les informations pertinentes, la portée limitée de cet article ne permet pas une telle discussion.

15. La limitation de l'espace ne nous permet pas d'aborder la notion de transnormativité. Pour plus d'informations, voir Johnson (2016).

16. Comme nous le rappelle Medina (2017: 256), la première formulation de la notion de «violence épistémique " était celle de Chakravorty Spivak (1988) dans son essai "Can the Subaltern Speak?» («Les subalternes peuvent-elles parler?»). Notre sous-titre mobilise cette formule. Effectivement, les PTVAD et les personnes vivant avec une démence peuvent parler, mais les formes de violence épistémiques font trop souvent qu'elles ne sont pas entendues. 


\section{RÉSUMÉS}

Les écrits portant sur les personnes âgées trans ont documenté quelques cas anecdotiques dans lesquels des personnes trans vivant avec une démence oublient leur transition et se réidentifient avec leur sexe assigné à la naissance (« détransition »). Les communautés trans et leurs allié-es encouragent les personnes trans à s'engager dans la planification de leur fin de vie, dont la préparation de documents juridiques précisant leurs souhaits quant à leur identité et à leur expression de genre en cas « d'incapacité » causée par une démence. Bien que de tels documents s'avèrent utiles, nous soutenons que la planification de fin de vie est souvent implicitement basée sur des hypothèses cisnormatives et cogninormatives (système normatif basé sur les capacités cognitives). Une telle planification repose sur une conception de l'identité de genre comme étant stable tout au long du parcours de vie ("post-transition ») et suppose que le soi « pré-démence » est mieux outillé pour prendre des décisions que le soi «dément». Nous concluons en encourageant, sur la base d'une approche intersectionnelle, trans-affirmative, crip-positive et âge-positive, le respect de l'agentivité des personnes trans vivant avec une démence.

Literature on the topic of trans older adults has documented a few anecdotal cases in which some trans people living with dementia forgot they transitioned and reidentified with their sex assigned at birth ("detransition"). Trans communities and their allies have encouraged trans people to engage in end-of-life planning, including the preparation of legal documents that state their wishes regarding gender identity and expression in the event of "incapacity" caused by dementia. While useful, we contend that end-of-life planning is often implicitly based on cisnormative and cogninormative (normative system based on cognitive abilities) assumptions. Such planning is founded on a stable notion of gender identity throughout the life course ("posttransition") and assumes that the pre-dementia self is better equipped to make decisions than the "demented" self. We conclude by encouraging, based on an intersectional, trans-affirmative, crip-positive, and age-positive approach, respect for the agency of trans people with dementia.

\section{INDEX}

Thèmes : Recherches

Keywords : trans and nonbinary people, dementia, end-of-life planning and documents, cisgenderism/transphobia, ableism/cogniticism, ageism, epistemic injustices

Mots-clés : personnes trans et non binaires, démence, planification et documents de fin de vie, cisgenrisme/transphobie, capacitisme/cogniticisme, âgisme, injustices épistémiques

\section{AUTEURS}

\section{ALEXANDRE BARIL}

Alexandre Baril (pronoms : il, lui), Ph.D. en études des femmes, est professeur agrégé à l'École de service social de l'Université d'Ottawa et se spécialise sur le genre, les sexualités, les identités de genre (trans) et le handicap. La formation interdisciplinaire d'Alexandre Baril combine dix années en philosophie/éthique, un doctorat en études des femmes, ainsi que deux stages postdoctoraux ; l'un en études féministes, des genres et des sexualités à Wesleyan University (Bourse CRSH) et l'autre en science politique à Dalhousie University (Bourse Izaak Walton Killam). Ses recherches intersectionnelles se situent à la croisée des études de genre, queer, 
trans, du handicap/crip/mad, de la gérontologie critique et de la suicidologie critique. Ses travaux récents se penchent sur les discours tenus sur le suicide et le suicide assisté au sein des mouvements et des études anti-oppression. Il a publié plusieurs articles dans des revues telles que Hypatia: Journal of Feminist Philosophy; Feminist Review; TSQ: Transgender Studies Quarterly; Sexualities; Atlantis: Critical Studies in Gender, Culture \& Social Justice; Frontiers: A Journal of Women Studies; Annual Review of Critical Psychology; DSQ: Disability Studies Quarterly; Journal of Literary \& Cultural Disability Studies; Canadian Journal of Disability Studies; Disability \& Society; Somatechnics; Canadian Social Work Review; Genre, sexualité \& société; Recherches féministes; Revue Jeunes et Société; Criminologie; Canadian Journal of Law \& Society; Philosophiques et Recherches sociologiques \& anthropologiques.

\section{MARJORIE SILVERMAN}

Marjorie Silverman (pronoms : elle), Ph.D. en service social, est professeure agrégée à l'École de service social de l'Université d'Ottawa. À partir de son expérience clinique, de perspectives critiques en gérontologie sociale et de méthodes qualitatives créatives, elle poursuit ses recherches sur des sujets liés à l'aide familiale, à la démence, aux lieux et aux espaces, à la sociologie du corps, et plus récemment, aux études trans et aux études de genre. Elle a publié des articles dans des revues comme Dementia, Journal of Aging Studies, Journal of Gerontological Social Work, International Journal of Care and Caring, Qualitative Social Work, Affilia, Canadian Journal on Aging, Canadian Journal of Law \& Society, et Sexualities.

\section{MARIE-CLAIRE GAUTHIER}

Marie-Claire Gauthier a œuvré cinq années comme travailleuse sociale dans le champ de la pauvreté avant d'entreprendre une carrière académique. Durant sa pratique, elle a été témoin de multiples façons dont les groupes marginalisés sont discrédités dans leur capacité à comprendre leurs réalités, leurs besoins et leurs droits et que conséquemment, les connaissances développées pour répondre à leurs besoins sont souvent construite par des groupes experts qui ont une certaine ignorance des vécus marginaux. Dans le cadre de son doctorat, dont elle est détentrice des bourses FRQSC et BÉSO, elle transpose ces réflexions de façon critique à la profession du travail social sous une perspective anti-oppressive et du concept des injustices épistémiques. Se situant elle-même en position de privilège par rapport à certains groupes, en tant que chercheuse appartenant à un groupe dominant, elle réfléchit aux différentes manières de faire de la recherche auprès de populations marginalisées. Elle cherche à comprendre les manières dont se construisent les savoirs en travail social et les incidences que ceux-ci ont sur l'élaboration des interventions, en explorant les questions de privilèges, d'oppressions et de pouvoir en travail social, notamment auprès des populations trans.

\section{MAUDE LÉVESQUE}

Maude Lévesque est une candidate au doctorat en service social à l'Université d'Ottawa où elle poursuit une spécialisation en gérontologie sociale sous une œillère représentationnelle. Elle a récemment obtenu son $\mathrm{PhD}$ en représentations sociales et communication auprès de l'Université de Rome (Sapienza) sur l'étude particulière de la détresse professionnelle des travailleurs sociaux canadiens en contexte de santé. Globalement, ses recherches se penchent sur les inégalités croissantes au sein de la population canadienne vieillissante par l'étude des services de santé et des soins de fin de vie, autant de la perspective des bénéficiaires de services que des pourvoyeurs de soins. 